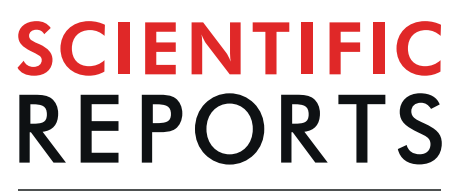

\title{
microRNA-670 modulates Igf2bp1 expression to regulate RNA methylation in parthenogenetic mouse embryonic development
}

\author{
Jindong $\mathrm{HaO}^{1,4}$, Haobo $\mathrm{Hu}^{1,4}$, Ziping Jiang ${ }^{2,4}$, Xianfeng $\mathrm{Yu}^{1}$, Chengshun $\mathrm{Li}^{1}$, Lin $\mathrm{Chen}^{1}$, \\ Yidan $\mathrm{Xia}^{2}$, Da Liu ${ }^{3 *}$ \& Dongxu Wang ${ }^{1 *}$
}

Aberrant epigenetic modification, including $N^{6}$-methylation of adenosine $(\mathrm{m} 6 \mathrm{~A})$, has been frequently reported in embryos derived from parthenogenetic activation (PA). However, the role of Igf $2 b p 1$ expression pattern in m6A modification and the mechanism through which Igf2bp1 function is regulated in PA embryos remains elusive. Therefore, in this study, using si-Igf2bp1 and betaine $(N, N, N$ trimethylglycine, a major methyl donor), we investigated the effect of Igf $2 b p 1$ expression in $\mathrm{m} 6 \mathrm{~A}$ modification on the development of PA embryos. The results indicated that the down-regulation of Igf $2 b p 1$ reduced the cleavage and blastula rates of PA embryos. Moreover, m6A expression level was markedly down-regulated following microinjection with si-lgf2bp1. However, the treatment with betaine could significantly restore the $\mathrm{m} 6 \mathrm{~A}$ level. Further bioinformatics analysis revealed $\lg f 2 b p 1$ as the putative target of microRNA 670 (miR-670). Thus, to confirm this finding, mimics and inhibitor of miR-670 were microinjected into PA embryos. The results demonstrated that miR-670 inhibitor augmented the expression of Igf $2 b p 1$ and rescued cleavage and blastula rates. In addition, the miR-670 inhibitor promoted the $\mathrm{m} 6 \mathrm{~A}$ expression level. TUNEL assay revealed a loss of expression of $\operatorname{lgf} 2 \mathrm{bp} 1$ induced cell apoptosis in PA embryos. Taken together, these results demonstrated that miR-670-3p functions as the regulator of Igf $2 b p 1$ expression and plays a crucial role in PA development through $\mathrm{m} 6 \mathrm{~A}$ modification.

Embryos derived from parthenogenetic (PA) activation comprise exclusively of maternal genomes and represent an important biological model for studies on epigenetic processes, including DNA methylation ${ }^{1}$. However, due to the absence of paternal gene expression and aberrant methylation profile, embryos generated from PA have a developmental failure ${ }^{2}$. Accumulating studies have also demonstrated abnormal DNA methylation in PA embryos; however, there is a paucity of studies related to the role of RNA methylation in PA embryos ${ }^{3,4}$.

$N^{6}$-methylation of adenosine $\left(N^{6}\right.$-methyladenosine or $\left.\mathrm{m}^{6} \mathrm{~A}\right)$, is the most abundant post-transcriptional modification of $\mathrm{RNA}^{5,6}$. An increasing number of studies indicate that $\mathrm{m}^{6} \mathrm{~A}$ plays a crucial role in several key developmental processes of the cell as well as regulates several aspects of RNA processing mechanisms that control gene expression in diverse physiological processes. Furthermore, the effects of $\mathrm{m}^{6} \mathrm{~A}$ on RNA are predominantly determined by the dynamic interplay between its methyltransferases ("writers"), binding proteins ("readers"), and demethylases ("erasers"). The insulin-like growth factor-2 mRNA-binding protein 1 (Igf2bpl) as novel $\mathrm{m}^{6} \mathrm{~A}$ "reader," recognizes $\mathrm{m}^{6} \mathrm{~A}$ sites on target mRNAs and regulates the mRNAs' fate ${ }^{7-9}$. Recent studies have identified $\operatorname{Igf} 2 \mathrm{bp} 1$ as a conserved 'oncogenic' $\mathrm{m}^{6} \mathrm{~A}$-reader which plays essential roles in carcinogenesis. Moreover, Igf $2 \mathrm{bp}$ proteins could enhance mRNAs stability and translation through m6A modification ${ }^{8}$. These pieces of evidence indicate that Igf $2 b p 1$ expression is associated with $\mathrm{m} 6 \mathrm{~A}$ modification and might have a role in PA embryonic development.

MicroRNAs (miRNAs) are endogenous small non-coding RNAs of approximately 22 nucleotides in length which are involved in the posttranscriptional regulation of gene expression by the degradation of their target

${ }^{1}$ Laboratory Animal Center, College of Animal Science, Jilin University, Changchun, China. ${ }^{2}$ Department of hand surgery, The First Hospital of Jilin University, Changchun, China. ${ }^{3}$ Department of Pharmacy, Changchun University of Chinese Medicine, Changchun, China. ${ }^{4}$ These authors contributed equally: Jindong Hao, Haobo Hu and Ziping Jiang. *email: liuda_1986@163.com; wang_dong_xu@jlu.edu.cn 


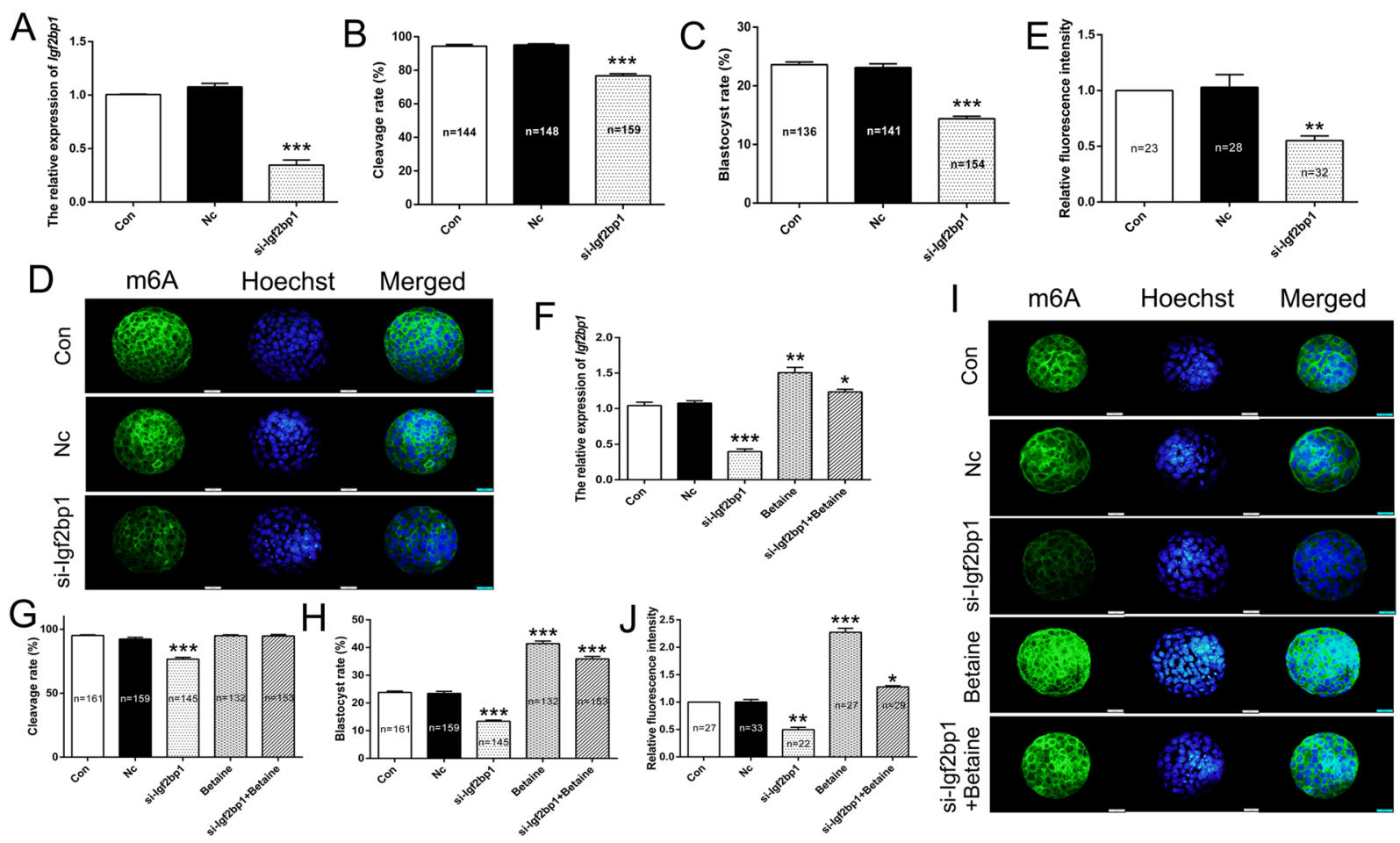

Figure 1. The expression of Igf $2 b p 1$ and m6A in PA embryos. Relative expression levels of Igf $2 b p 1$ as analyzed by qRT-PCR following micro-injection with si-Igf2bp1 in PA embryos (A). (A) Statistical analyses of cleavage (B) and blastula $(C)$ rates following micro-injection with si-Igf2bp1. Immunofluorescence localization of m6A following micro-injection with si-Igf2bp1 (D). (D) Statistical analyses of fluorescence intensity (E). (E) Relative expression levels of $I g f 2 b p 1$ as analyzed by qRT-PCR after treatment with betaine in PA embryos (F). (F)

Statistical analyses of cleavage $(\mathbf{G})$ and blastula $(\mathbf{H})$ rates following treatment with betaine. Immunofluorescence localization of m6A following treatment with betaine (I). (I) Statistical analyses of fluorescence intensity (J). (J) The data are represented as the mean $\pm \mathrm{SD}(\mathrm{n}=3)$. Green, indicates m6A. Blue, indicates Hoechst. The bar $=20$ $\mu \mathrm{m} .{ }^{*} p<0.05, * * p<0.01$ and $* * * p<0.005$ indicate statistically significant differences.

mRNAs and/or inhibiting their translation. Recently, miR-670 has been reported to target INTS4 in the glomeruli of NZB/W F1 mice with lupus nephritis ${ }^{10}$. Moreover, miR-670 has been identified to be associated with endometrial cancer by targeting $\mathrm{KCNS}^{11}$. Betaine ( $N, N, N$-trimethylglycine), as a major methyl donor in the cycle of one-carbon metabolism, has been extensively used to treat pre-implantation embryos to improve the development of embryos ${ }^{12}$. Recent studies have reported that betaine has a role in mouse oocyte meiotic maturation through choline dehydrogenase ${ }^{13}$. Furthermore, betaine can efficiently restore the development of embryos injured by ethanol through a global level of genome DNA methylation in the mouse embryo ${ }^{14}$. In the present study, the expression levels of Igf $2 b p 1$ and m6A were determined using qRT-PCR during early PA embryonic development following treatment with si-RNA and betaine. Overall, the role of $\operatorname{Ig} f 2 b p 1$ in m6A modification was analyzed in the development of PA embryos.

\section{Results}

Knockdown of Igf2bp1 expression attenuated global mRNA m6A expression levels. To investigate the role of Igf $2 b p 1$ during embryonic development, si-Igf $2 \mathrm{bp} 1$ and negative control siRNA were microinjected into a zygote. The expression levels of $I g f 2 b p 1 \mathrm{mRNA}$ were analyzed using qRT-PCR. The results indicated that Igf $2 b p 1$ expression levels were significantly downregulated in PA embryos injected with si-Igf2bpl compared with those in the negative control siRNA-injected embryos (Nc) or non-injected embryos (Con) (Fig. 1A). Moreover, knockdown of Igf2bp1 expression significantly suppressed the cleavage and blastula rates in PA embryos (Fig. 1B,C). Furthermore, m6A expression level was analyzed using immunofluorescence (IF). The results indicated that m6A expression was significantly suppressed in PA embryos injected with si-Igf $2 \mathrm{bp} 1 \mathrm{com}-$ pared with the Nc group and Con group (Fig. 1D,E). Collectively, these findings suggested that Igf $2 b p 1$ markedly affected the global mRNA levels of m6A and Igf $2 b p 1$ expression were associated with PA embryo development.

Betaine up-regulated m6A expression and promoted PA embryonic development. To investigate whether the blastocyst rate was associated with Igf2bp1 expression, the betaine was applied to the culture of PA embryos. qRT-PCR results revealed that betaine significantly upregulated the expression of Igf $2 b p 1$ following transfection with si-Igf2bp1 (Fig. 1F). Furthermore, the cleavage rate was restored after treatment with betaine (Fig. 1G). Besides, the blastula rate was also noticeably increased in the si-Igf2bp1 group after treatment with betaine (Fig. $1 \mathrm{H})$. In addition, the results of IF showed that betaine significantly up-regulated the expression of 

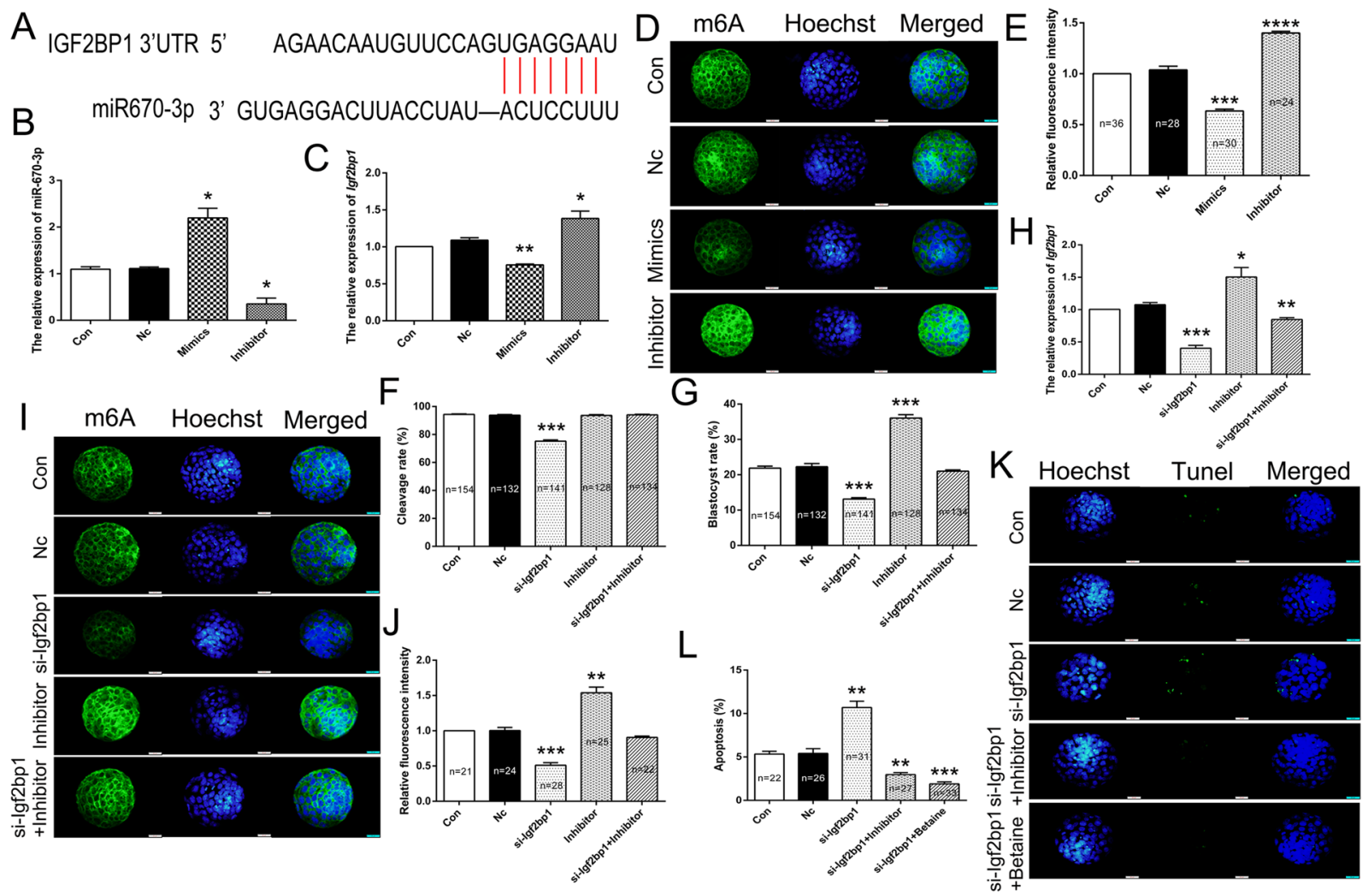

Figure 2. The expression of Igf2bp1 was regulated by miRNA-670. Schematic representations of target sites of miRNA-670 within 3'UTR of Igf2bp1 mRNA (A). (A) Relative expression levels of miRNA-670 (B) and Igf2bp1 (C) as analyzed by qRT-PCR. Immunofluorescence localization of m6A following micro-injection with miRNA670 mimics and inhibitor (D). (D) Statistical analysis of fluorescence intensity (E). (E) Statistical analysis of cleavage $(\mathbf{F})$ and blastula $(\mathbf{G})$ rates following micro-injection with a miRNA-670 inhibitor in PA embryos. Relative expression levels of Igf $2 b p 1$ as analyzed by qRT-PCR $(\mathbf{H})$. Immunofluorescence localization of m6A following micro-injection with miRNA-670 inhibitor and si-Igf2bp1 (I). (I) Statistical analysis of fluorescence intensity $(\mathrm{J})$. (J) Green, indicates m6A. Blue, indicates Hoechst in m6A IF staining. TUNEL assay to analyze the cell apoptosis in PA embryos (K). (K) Statistical analysis of cell apoptosis (L). (L) Blue, indicates Hoechst. Green, indicates Tunel. $* p<0.05, * * p<0.01, * * * p<0.005$ and $* * * * p<0.001$ indicate statistically significant differences.

m6A (Fig. 1I,J). Together, these results indicated that increased expression levels of Igf2bpl and global expression of m6A promoted PA embryonic development following treatment with betaine.

miR-670-3p regulated Igf 2 bp 1 expression in PA embryos apoptosis. To investigate the post-transcriptional regulation of $\operatorname{Ig} f 2 b p 1$, potential miRNAs targeting $\operatorname{Ig} f 2 b p 1$ were identified using bioinformatics tools (Fig. 2A). The qRT-PCR analysis revealed that miR-670-3p could negatively regulate the expression of Igf2bpl (Fig. 2B,C). Besides, IF results indicated that inhibitors of miR-670-3p markedly augmented m6A expression levels compared to the Nc group (Fig. 2D,E). Moreover, the cleavage and blastula rates were analyzed in the si-Igf $2 \mathrm{bp} 1$ group following transfection with an inhibitor of miR-670-3p. The results revealed that the miR-670-3p inhibitor could restore the cleavage and blastula rates in PA embryos (Fig. 2F,G). To verify whether $\operatorname{Igf} 2 b p 1$ is a direct target of miR-670-3p, the expression of Igf $2 b p 1$ in the si-Igf $2 \mathrm{bp} 1$ group was analyzed following treatment with miR-670-3p inhibitor. The qRT-PCR analysis indicated that Igf2bpl expression was markedly up-regulated in the si-Igf2bp1 group following treatment with miR-670-3p inhibitor (Fig. $2 \mathrm{H}$ ). Furthermore, IF data suggested that miR-670-3p inhibitor could increase m6A expression levels in the si-Igf2bp1 group (Fig. 2I,J). Besides, the TUNEL assay revealed that treatment with miR-670-3p inhibitor noticeably reduced the number of apoptotic cells (Fig. 2K,L). Collectively, these results indicated that miR-670-3p modulated the expression of Igf $2 b p 1$ and influenced m6A expression levels.

\section{Discussion}

Our previous study suggested that global mRNA m6A expression levels are associated with PA embryonic development $^{15}$. Therefore, to further elucidate the underlying molecular mechanism of m6A modification in mice parthenogenesis, the expression of Igf $2 b p 1$ was analyzed at the cleavage and blastocyst stages of the PA embryos in the present study. The results indicated that an increased rate of development failure is associated with reduced m6A expression levels in PA embryos. These findings confirmed that m6A expression level was crucial for 
embryonic development which affects RNA stability. Moreover, Igf2 $b p 1$ exhibits a role in recognizing and binding to the m6A writing sites of targeted mRNAs ${ }^{16}$. This implied that reduced expression of Igf $2 b p 1$ might inhibit m6A level which is in concordance with our data. Thus, the findings of the present study suggested that the Igf2bpl exhibits the ability to regulate PA embryonic development through m6A modification.

Further, to investigate the post-transcriptional regulation of $\operatorname{Ig} f 2 b p 1$, potential miRNAs targeting Igf $2 b p 1$ were identified using bioinformatics tools. To confirm that Igf $2 b p 1$ is the target of miR-670, mimics, and miR-670 inhibitor was micro-injected in the PA embryos. The results indicated that in the presence of the miR-670 inhibitor, Igf $2 b p 1$ expression was markedly upregulated and the expression levels of $\mathrm{m} 6 \mathrm{~A}$ were considerably promoted as compared with the si-Igf $2 \mathrm{bp} 1$ group. Moreover, the inhibitor of miR-670 rescued the development of embryos which was suppressed by the loss of expression of Igf2 $b p 1$. Indeed, several miRNAs have been recognized to play a crucial role in the development of PA embryos ${ }^{17,18}$. Thus, considering these pieces of evidence, we speculated that m6A methylation level is associated with miR-670 and $\operatorname{Igf} 2 \mathrm{bp} 1$ and may represent crucial factors for developmental failure.

Furthermore, treatment with betaine promotes DNA methylation ${ }^{19,20}$. Moreover, there is evidence that betaine improves embryo development in mice ${ }^{13}$. However, there is a paucity of literature regarding the role of m6 $\mathrm{A}$ modification following treatment with betaine. Our result suggested that the m6A level was up-regulated following treatment with betaine. Furthermore, betaine rescued Igf $2 b p 1$ expression and promoted the development of PA embryos in the si-Igf $2 \mathrm{bp} 1$ group. These findings indicated that m6A modification which was regulated by betaine exhibited a crucial role in PA embryonic development. In addition, compared to the si-Igf 2 bp 1 group, treatment with betaine and miR-670 inhibitor markedly inhibited apoptosis. Previous studies suggested that Igf $2 b p 1$ overexpression suppressed apoptosis in HTR-8/SVneo cells which were consistent with our results ${ }^{21}$. Thus, Igf2bp1 expression exhibits a crucial role in cell apoptosis in PA embryonic development.

\section{Conclusion}

The findings of this study suggested that loss of expression of Igf $2 b p 1$ markedly suppressed PA embryonic development and m6A levels. Furthermore, our data also demonstrated that miR-670 inhibitor augmented the expression of $\operatorname{Igf} 2 \mathrm{bp} 1$ and rescued cleavage and blastula rates. Besides, betaine could enhance the m6 A methylation level in PA embryos. In conclusion, miR-670 functions as the regulator of Igf2bpl expression and plays a crucial role in PA development through m6A modification.

\section{Methods}

Ethics statement. The animals were cared for in accordance with the Guide for the care and use of laboratory animals in China. All experimental procedures were approved by the Animal Care and Use Committee of Jilin University, Changchun, China (Grant No. 201706005).

Production of PA embryos. The female ICR mice (6-8 weeks old) were acquired from the School of Medical Science, Jilin University. Superovulation was induced in female mice by an intraperitoneal administration of 10 IU pregnant mare's serum gonadotropin (PMSG; Merck Millipore) and was injected with 10 IU human chorionic gonadotropin (hCG; Sigma-Aldrich, St. Louis, MO, USA)) $48 \mathrm{~h}$ later. PA embryos production was performed according to the procedure described by us previously. Briefly, the oviducts of female mice were carefully removed, and cumulus-oocyte complexes (COCs) were extracted from the oviducts as unfertilized oocytes after post-PMSG and -hCG injection. Oocytes were then denuded from their cumulus cells by briefly exposing the oocytes at the metaphase stage of the second meiotic division (MII) to a serum-free medium comprising hyaluronidase (Sigma). The oocytes were then treated with a calcium ionophore (ionomycin calcium salt; Sigma) for $5 \mathrm{~min}$. Parthenogenetic activation was achieved by incubating the unfertilized oocytes in M16 medium supplemented with 6-DMAP ( $2 \mathrm{mmol} / \mathrm{l}$; Sigma) for $4 \mathrm{~h}$. Subsequently, these unfertilized oocytes were transferred to the fresh M16 medium. PA activation was established by the presence of two pronuclei, which developed to the two-celled stage. The embryo culture was conducted until the blastocyst stage.

Microinjection of microRNA and siRNA into the zygote. Before microinjection, oocytes were cultured to metaphase-II (MII) with completed parthenogenetic activation. Microinjections for mouse miR-670 (mmu-miR-670-3p) and si-Igf2bp1 into the cytoplasm of zygote were performed using an Eppendorf Cell Tram Vario system (Eppendorf, Hamburg, German) and a Nikon TE2000-U inverted microscope (Nikon, Tokyo, Japan). Similarly, the siRNA negative control and miRNA negative control was microinjected into zygote to serve as the negative control, while non-injected zygote was used as the normal control. The final concentration of the control or miR- 670 or si-Igf $2 \mathrm{bp} 1$ was $20 \mu \mathrm{M}$. The injected zygotes were cultured M16 medium until the blastocyst stage. The sequence of si-Igf $2 \mathrm{bp} 1$ was $\left(5^{\prime}\right.$ to $\left.3^{\prime}\right)$ : TTTACTTCCTCCTTGGGACTT; the sequence of mmu-miR-670-3p mimics was ( $5^{\prime}$ to $\left.3^{\prime}\right)$ : UUUCCUCAUAUCCAUUCAGGAGU; the sequence of mmu-miR670-3p inhibitor was $\left(5^{\prime}\right.$ to $\left.3^{\prime}\right)$ : ACACUCCUGAAUGGAUAUGAGGAAA; the sequence of miRNA Nc was $\left(5^{\prime}\right.$ to $3^{\prime}$ ): UUCUCCGAACGUGUCACGU.

Gene expression analysis. The gene expression analysis was performed as described previously ${ }^{22}$. Briefly, the total RNA was extracted from embryos of each group $(n=50)$. Quantitative real-time PCR (qRT-PCR) was used to analyze the expression of Igf2bp1. qRT-PCR was performed using the following two-step cycling program: initial denaturation at $95^{\circ} \mathrm{C}$ for $3 \mathrm{~min}$, followed by 40 cycles of denaturation at $95^{\circ} \mathrm{C}$ for $10 \mathrm{~s}$, annealing at $60^{\circ} \mathrm{C}$ for $15 \mathrm{~s}$, and extension at $72^{\circ} \mathrm{C}$ for $30 \mathrm{~s}$. The $2^{-\Delta \Delta \mathrm{CT}}$ method was used to determine the relative gene expression, which was normalized to the expression of endogenous control, Gapdh. The experiments were performed at least in triplicate. The primer sequences used in the qRT-PCR analysis were as follows: Igf $2 b p 1$ forward, 5' -ATCGGAGCTGAGGTGGAATA-3'; reverse, 5'-CTCGGGGAAAGTAGAACTGC-3'; Gapdh forward, 5'-AGGTCGGTGTGAACGGATTTG-3'; reverse, 5'-TGTAGACCATGTAGTTGAGGTCA-3'. 
Immunofluorescence staining. The mouse immunofluorescence (IF) staining was performed on oocytes/ embryos, as described previously ${ }^{15}$. Briefly, using Tyrode's Solution (Jisskang, China), the thinning of zona pellucida was performed. Then, embryos were fixed with $4 \%$ paraformaldehyde in phosphate-buffered saline (PBS) for $30 \mathrm{~min}$ at room temperature (RT). Following fixation, the embryos were permeabilized with $0.2 \%$ Triton X-100 in PBS for $30 \mathrm{~min}$. They were then blocked in PBS containing $1 \%$ bovine serum albumin (BSA) for $1 \mathrm{~h}$. Next, the embryos were probed with m6A (1:500, Abcam) antibodies and incubated at $4{ }^{\circ} \mathrm{C}$ overnight. After incubation with the primary antibody, embryos were washed three times with PBS followed by incubation with Alexa Fluor 488 conjugated secondary (anti-rabbit) antibodies for $1 \mathrm{~h}$ at RT. The embryos were stained with $10 \mathrm{ng} / \mathrm{ml}$ Hoechst 33342 (Thermo Scientific) for 15-20 min. Slides were mounted using an anti-fade mounting medium (BOSTER, China). Images were acquired analyzed using a fluorescence microscope $1-2 \mathrm{~h}$ after staining. The exposure time of IF staining was $10 \mathrm{~ms}$ for Hoechst and $70 \mathrm{~ms}$ for $\mathrm{m} 6 \mathrm{~A}$. The average fluorescence intensities were analyzed using ImageJ analysis software ${ }^{23}$.

TUNEL assay. Briefly, the blastocysts were fixed with $4 \%$ paraformaldehyde for $1 \mathrm{~h}$ at RT. After fixation, the blastocysts were permeabilized with $0.1 \%$ Triton X-100 for $1 \mathrm{~h}$ at $37^{\circ} \mathrm{C}$. The blastocysts were blocked with PBS containing $1 \%$ BSA in the dark for $1 \mathrm{~h}$ at $37^{\circ} \mathrm{C}$ with TdT and fluorescein-conjugated dUTP (In Situ Cell Death Detection kit; Roche, Germany). The blastocysts were stained with $10 \mu \mathrm{g} / \mathrm{mL}$ Hoechst 33342 for $15 \mathrm{~min}$. Slides were mounted using an anti-fade mounting medium (BOSTER, China). The blastocysts were mounted on a coverslip and a glass slide using an antifade mounting medium (BOSTER, China). Images were acquired using a fluorescence microscope $1-2 \mathrm{~h}$ after staining and the cell apoptotic rates were analyzed using ImageJ software.

Statistical analysis. TargetScan program (www.targetscan.org) was used to predict miRNAs (miR-670) binding sites on mRNA of Igf $2 b p 1$. Data are presented as mean \pm SD of at least three replicates. Differences between groups were assessed using Student's $t$-test (Unpaired $t$-test). Statistical analyses were performed with GraphPad Prism 5.0 (GraphPad Software, Inc., San Diego, CA). A p-value of $<0.05$ was considered statistically significant.

Received: 14 November 2019; Accepted: 3 March 2020;

Published online: 16 March 2020

\section{References}

1. Park, C. H., Kim, H. S., Lee, S. G. \& Lee, C. K. Methylation status of differentially methylated regions at Igf2/H19 locus in porcine gametes and preimplantation embryos. Genomics 93, 179-186, https://doi.org/10.1016/j.ygeno.2008.10.002 (2009).

2. Zhu, J. et al. In vitro and in vivo developmental competence of ovulated and in vitro matured porcine oocytes activated by electrical activation. Cloning and stem cells 5, 355-365, https://doi.org/10.1089/153623003772032853 (2003).

3. Park, C. H. et al. Analysis of Imprinted Gene Expression in Normal Fertilized and Uniparental Preimplantation Porcine Embryos. PloS one 6, https://doi.org/10.1371/journal.pone.0022216 (2011).

4. Horii, T., Kimura, M., Morita, S., Nagao, Y. \& Hatada, I. Loss of genomic imprinting in mouse parthenogenetic embryonic stem cells. Stem cells 26, 79-88, https://doi.org/10.1634/stemcells.2006-0635 (2008).

5. Geula, S. et al. Stem cells. m6A mRNA methylation facilitates resolution of naive pluripotency toward differentiation. Science 347, 1002-1006, https://doi.org/10.1126/science.1261417 (2015).

6. Dominissini, D. et al. Topology of the human and mouse m6A RNA methylomes revealed by m6A-seq. Nature 485, 201-206, https:// doi.org/10.1038/nature11112(2012).

7. Nguyen, L. H. et al. Lin28b Is Sufficient to Drive Liver Cancer and Necessary for Its Maintenance in Murine Models. Cancer cell 26, 248-261, https://doi.org/10.1016/j.ccr.2014.06.018 (2014).

8. Huang, H. et al. Recognition of RNA N(6)-methyladenosine by IGF2BP proteins enhances mRNA stability and translation. Nature cell biology 20, 285-295, https://doi.org/10.1038/s41556-018-0045-z (2018).

9. Wang, X., He, C. \& Reading, R. N. A. methylation codes through methyl-specific binding proteins. Rna Biol. 11, 669-672, https:// doi.org/10.4161/rna.28829 (2014)

10. Tian, S. et al. Microarray expression and functional analysis of circular RNAs in the glomeruli of NZB/W F1 mice with lupus nephritis. Experimental and therapeutic medicine 18, 2813-2824, https://doi.org/10.3892/etm.2019.7901 (2019).

11. Xu, X. et al. miRNA-mRNA Associated With Survival in Endometrial. Cancer. Frontiers in genetics 10, 743, https://doi.org/10.3389/ fgene.2019.00743 (2019).

12. Karunamuni, G. et al. Supplementation with the Methyl Donor Betaine Prevents Congenital Defects Induced by Prenatal Alcohol Exposure. Alcoholism, clinical and experimental research 41, 1917-1927, https://doi.org/10.1111/acer.13495 (2017).

13. McClatchie, T. et al. Betaine is accumulated via transient choline dehydrogenase activation during mouse oocyte meiotic maturation. The Journal of biological chemistry 292, 13784-13794, https://doi.org/10.1074/jbc.M117.803080 (2017).

14. Zhang, D. et al. Supplement of Betaine into Embryo Culture Medium Can Rescue Injury Effect of Ethanol on Mouse Embryo Development. Scientific reports 8, 1761, https://doi.org/10.1038/s41598-018-20175-w (2018).

15. Hao, J. et al. [PROVISIONAL] The perturbed expression of m6A in parthenogenetic mouse embryos. Genetics and molecular biology, https://doi.org/10.1590/1678-4685-GMB-2018-0212 (2019).

16. Zhao, B. S. et al. m(6)A-dependent maternal mRNA clearance facilitates zebrafish maternal-to-zygotic transition. Nature 542, 475-478, https://doi.org/10.1038/nature21355 (2017).

17. Zhang, Z., Zhuang, L. \& Lin, C. P. Roles of MicroRNAs in Establishing and Modulating Stem Cell Potential. International journal of molecular sciences 20, https://doi.org/10.3390/ijms20153643 (2019).

18. Yang, W. et al. High-throughput transcriptome-Seq and small RNA-Seq reveal novel functional genes and microRNAs for early embryonic arrest in humans. Gene 697, 19-25, https://doi.org/10.1016/j.gene.2018.12.084 (2019).

19. Zhao, N., Yang, S., Sun, B., Feng, Y. \& Zhao, R. Maternal betaine protects rat offspring from glucocorticoid-induced activation of lipolytic genes in adipose tissue through modification of DNA methylation. European journal of nutrition, https://doi.org/10.1007/ s00394-019-02025-1 (2019).

20. Mahmoud, A. M. \& Ali, M. M. Methyl Donor Micronutrients that Modify DNA Methylation and Cancer Outcome. Nutrients 11, https://doi.org/10.3390/nu11030608 (2019).

21. Guo, L., Liu, Y., Guo, Y., Yang, Y. \& Chen, B. MicroRNA-423-5p inhibits the progression of trophoblast cells via targeting IGF2BP1. Placenta 74, 1-8, https://doi.org/10.1016/j.placenta.2018.12.003 (2018). 
22. Gao, W. et al. Ascorbic acid improves parthenogenetic embryo development through TET proteins in mice. Biosci. Rep. 39, https:// doi.org/10.1042/BSR20181730 (2019).

23. Zaitseva, I., Zaitsev, S., Alenina, N., Bader, M. \& Krivokharchenko, A. Dynamics of DNA-demethylation in early mouse and rat embryos developed in vivo and in vitro. Molecular reproduction and development 74, 1255-1261, https://doi.org/10.1002/mrd.20704 (2007).

\section{Acknowledgements}

This work was supported by grants from the National Natural Science Foundation of China under Grant 31601003; the China Postdoctoral Science Foundation under Grant 2018T110250 and 2016M601384; the Fundamental Research Funds for the Central Universities under Grant 2019JCKT-70; and the Jilin Scientific and Technological Development Program under Grant 20190103071JH, 20180101254JC, and 0170623093-TC.

\section{Author contributions}

Dongxu Wang designed the experiments and prepared the manuscript. Jindong Hao, Haobo Hu, and Chengshun Li performed cell experiments and gene expression analyses. Da Liu, Ziping Jiang and Xianfeng Yu donated reagents and materials required. Lin Chen, Yidan Xia carried out animal experiments. Da Liu analyzed the data and prepared figures. All authors have read and approved the final manuscript.

\section{Competing interests}

The authors declare no competing interests.

\section{Additional information}

Supplementary information is available for this paper at https://doi.org/10.1038/s41598-020-61816-3.

Correspondence and requests for materials should be addressed to D.L. or D.W.

Reprints and permissions information is available at www.nature.com/reprints.

Publisher's note Springer Nature remains neutral with regard to jurisdictional claims in published maps and institutional affiliations.

Open Access This article is licensed under a Creative Commons Attribution 4.0 International License, which permits use, sharing, adaptation, distribution and reproduction in any medium or format, as long as you give appropriate credit to the original author(s) and the source, provide a link to the Creative Commons license, and indicate if changes were made. The images or other third party material in this article are included in the article's Creative Commons license, unless indicated otherwise in a credit line to the material. If material is not included in the article's Creative Commons license and your intended use is not permitted by statutory regulation or exceeds the permitted use, you will need to obtain permission directly from the copyright holder. To view a copy of this license, visit http://creativecommons.org/licenses/by/4.0/.

(c) The Author(s) 2020 